\title{
One pot synthesis of some new 2-hydrazino-[1,3,4]thiadiazepino $[7,6-b]$ quinolines under microwave irradiation conditions
}

\author{
M. Raghavendra, H. S. Bhojya Naik* and B. S. Sherigara \\ Dept. of PG Studies and Research in Industrial Chemistry, School of Chemical Sciences, \\ Kuvempu University, Shankaraghatta- 577 451, Karnataka, India \\ E-mail: hsbnaik@,rediffmail.com
}

\begin{abstract}
An efficient and convenient procedure has been developed for the synthesis of 2-hydrazino$[1,3,4]$ thiadiazepino[7,6-b]quinolines (2a-h) in good yields. They have been achieved by the reaction between corresponding 2-chloro-3-formyl-quinoline and carbidimide in specially desighned microwave oven for organic synthesis in unsealed borosil vessel in presence of $p$ $\mathrm{TsOH}$ and dimethylformamide. The structure of new compounds has been evaluated on the basis of analytical, IR, ${ }^{1} \mathrm{H}$ NMR and mass spectral data.
\end{abstract}

Keywords: Quinoline, thiadiazepino[7,6- $b]$ quinolines, microwave irradiation

\section{Introduction}

Several polycyclic analogues of natural or synthetic antitumor agents are well known, and have attracted considerable interest because of their significant anticancer activity. ${ }^{1}$ There is a evidence that anticancer activity is due to the intercalation between the drugs and the base pairs of DNA and interference with normal functioning of the enzyme topoisomerase II which is involved in the breaking and releasing of DNA strands. ${ }^{2}$ The intercalative binding of these drugs is due to the presence of planar linearly fused tri and tetracyclic systems ${ }^{3}$. Particularly, five and six membered heterocyclic compounds containing one or two heteroatoms fused to a quinoline ring in linear fashion are found in natural products as well as in synthetic compounds of biological interest have antitumor and anticancer properties. ${ }^{4,5}$ They are also known to exhibit antiallergenic, ${ }^{6}$ antifungal, ${ }^{7}$ hypocholesterolemic, hypolemic, ${ }^{8}$ antibacterial, ${ }^{9}$ and antiviral $^{10}$ properties.

On the other hand, the synthesis of thiadiazepines has attracted the attention of chemists because they are associated with various types of biological activities such as antimicrobial, ${ }^{11}$ antiviral, and anthelmintic activities ${ }^{12}$. Also, $[1,3,4]$ thiadiazole derivatives shows antibacterial, ${ }^{13-}$ 14 antiinflammatory, ${ }^{15} \mathrm{CNS}$ depressant action, ${ }^{16}$ and moderate anti-malarial and anti-tumor activities. ${ }^{17}$ To the best of our knowledge only a few reports are available for the synthesis, and 
pharmacological activity of 1,2,4-thiadiazepines, 1,2,7-thiadiazepines, 1,3,4-thiadiazepines, and 1,4,5-thiadiazepines. ${ }^{18-19}$

Microwave heating has emerged as a powerful technique to promote a variety of chemical reactions due to the short reaction time and the operational simplicity. So a number of research papers have appeared proving the synthetic utility of MORE (Microwave-induced Organic Reaction Enhancement) chemistry in organic synthesis. ${ }^{20-23}$ A recent literature survey reveals that thiadiazepines were obtained by a [6-1] cyclocondensation reaction, ${ }^{24}$ ring formation by the reaction of thioimidates and diimines with dihalo-containing cyclization agents, ${ }^{25-26}$ and other miscellaneous methods. ${ }^{27}$ In view of the above findings and in continuation of our work on microwave assisted synthesis of biologically important condensed heterocycles ${ }^{28}$ herein we wish to report a simple, convenient microwave assisted synthesis of 2-hydrazino$[1,3,4]$ thiadiazepino[7,6- $b]$ quinolines (2a-h) in presence of $p$-TsOH catalyst from the reaction of 2-chloro-3-formyl-quinoline and carbidimide in shorter time with good yield (Scheme-1).<smiles>[R]c1cc2cc(C(=O)c3ccccc3)c(Cl)nc2c([R3])c1[R2]</smiles>

(1a-h)<smiles>NNC(=S)NN</smiles>

(2a) $\mathrm{R}_{1}=\mathrm{R}_{2}=\mathrm{R}_{3}=\mathrm{H}$

(2b) $\mathrm{R}_{1}=\mathrm{R}_{3}=\mathrm{H}, \mathrm{R}_{2}=\mathrm{CH}_{3}$

(2c) $\mathrm{R}_{1}=\mathrm{CH}_{3}, \mathrm{R}_{2}=\mathrm{R}_{3}=\mathrm{H}$

(2d) $\mathrm{R}_{1}=\mathrm{R}_{2}=\mathrm{H}, \mathrm{R}_{3}=\mathrm{CH}_{3}$

(2e) $\mathrm{R}_{1}=\mathrm{Cl}, \mathrm{R}_{2}=\mathrm{R}_{3}=\mathrm{H}$

(2f) $\mathrm{R}_{1}=\mathrm{R}_{3}=\mathrm{H}, \mathrm{R}_{2}=\mathrm{OCH}_{3}$

(2g) $\mathrm{R}_{1}=\mathrm{R}_{2}=\mathrm{H}, \mathrm{R}_{3}=\mathrm{OCH}_{3}$

(2h) $\mathrm{R}_{1}=\mathrm{OCH}_{3}, \mathrm{R}_{2}=\mathrm{R}_{3}=\mathrm{H}$<smiles>[R]c1cc2cc(/C=N\NC(=S)NN)c(Cl)nc2c([R])c1[R]</smiles><smiles>[R]c1cc2cc3c(nc2c([R3])c1[R2])SC(NN)=NN=C3</smiles>

(2a-h)

\section{Scheme 1}

\section{Results and Discussion}

Generally, planar fused condensed quinolines containing one or two hetero atoms found to have valuable pharmacological activities as mentioned earlier, and therefore, they are useful 
compounds in medicinal research. Hence, in continuation of research work on developing new quinoline containing heterocycles due to their significant biological activities, it appeared expedient to synthesize a series of condensed and appropriately funtionalised 2hydrazino[1,3,4] thiadiazepino[7,6- $b]$ quinolines in the present study. The starting compound 2chloro-3-formyl quinolines, (1a-h) were prepared according to the literature method ${ }^{29}$ The 2hydrazino-[1,3,4]thiadiazepino[7,6-b]quinoline (2a-h) were obtained in one pot by the cyclisation of 2-chloro-3-formylquinolines with carbidimide in presence of $p$-TsOH catalyst and DMF under microwave irradiation in good yields.

This method provides high yield of products in 5-6 min making it a useful method for the synthesis of condensed thiadiazepino quinolines.

The reaction proceeded through the intermediate Schiff base which was formed by the condensation of aldehyde group of quinoline and amine group of carbidimide followed by the intramolecular nucleophilic substitution of chlorine at C-2 of quinoline ring. The structure of the compounds was confirmed on the basis of elemental analysis and spectral data (experimental section). As an example, the IR (KBr) spectrum of the compound 2a showed an absence of $\mathrm{CHO}$ stretching frequency at $1670 \mathrm{~cm}^{-1}$ which appeared in the 2-chloro-3-formylquinoline 1a, the ${ }^{1} \mathrm{H}$ NMR (DMSO- $\mathrm{d}_{6}$ ) spectrum of $\mathbf{2 a}$ in addition to aromatic protons resonated between $\delta$ 6.9-7.9 ppm (6H) exhibited a singlet at $\delta 4.4 \mathrm{ppm}$ corresponding to $-\mathrm{NH}_{2}$ protons, broad singlet at $\delta 8.2 \mathrm{ppm}$ for $-\mathrm{NH}$ proton indicated the attachment of the reactive partner to the quinoline substrate. Finally, the structure was confirmed by its mass spectrum through the appearance of a molecular ion peak at m/z $243\left(\mathrm{M}^{+}\right)$. The obtained elemental analysis values are in agreement with theoretical data. We synthesized seven more title compounds, which exhibited similar spectral characteristics.

\section{Conclusions}

In conclusion, a simple efficient and environmentally benign method has been developed for the synthesis of 2-hydrazino-[1,3,4]thiadiazepino[7,6- $b$ ]quinolines under microwave irradiation conditions in presence of $p$ - $\mathrm{TsOH}$. This microwave irradiation method is superior from the view of yield and reaction time compared to the conventional (thermal) method.

\section{Experimental Section}

\section{General microwave procedure for the synthesis of substituted 2-hydrazino $[1,3,4]$ thiadiazepino[7,6-b]quinolines (2a-h)}

To a mixture of substituted quinoline 1a $(0.764 \mathrm{~g}, 0.004 \mathrm{~mol})$ and carbidimide $(0.530 \mathrm{~g}, 0.005$ mol), catalytic amount of $p$-TsOH and anhydrous dimethylformamide $(8 \mathrm{ml})$ were added and the contents were irradiated under microwave oven for about 4 minutes at an interval of $1 \mathrm{~min}$ 
at $160 \mathrm{~W}$. The completion of reaction was monitored by TLC, the product $\mathbf{2 a}$ was poured into ice-cold water, stirred well, filtered, dried and recrystallised from aqueous DMF. The same procedure was used for the synthesis of $(\mathbf{2} \mathbf{b}-\mathbf{h})$

\section{General conventional procedure for the synthesis of substituted 2-hydrazino} $[1,3,4]$ thiadiazepino[7,6-b]quinolines (2a-h)

Mixture of substituted quinoline 1a $(0.764 \mathrm{~g}, 0.004 \mathrm{~mol})$, carbidimide $(0.530 \mathrm{~g}, 0.005 \mathrm{~mol})$, catalytic amount of $p$-TsOH and $30 \mathrm{ml}$ absolute ethanol were taken in $100 \mathrm{ml}$ round bottom flask, kept for reflux for about 6 hours after the completion of the reaction confirmed by TLC, reaction mixture was concentrated then poured into ice cold water. The obtained greenish yellow colour solid was filtered washed with water then recrystallised from aqueous DMF. The same procedure was used for the synthesis of (2b-h).

2-Hydrazino[1,3,4]thiadiazepino[7,6-b]quinoline (2a). Irradiation time: 4 minutes, Yellow colour solid. Yield: $90 \%(\mathrm{MW})$, refluxed time 6 hours $69 \%$ (Conventional). Mp: $172-174{ }^{\circ} \mathrm{C}$. IR $(\mathrm{KBr}) \mathrm{cm}^{-1} 3340,3155,3300 .{ }^{1} \mathrm{H}$ NMR (400 MHz, DMSO-d 6 ) 4.4 (s, 2H, NH ), 8.2 (s, 1H, NH), 6.9-7.9 (m, 6H, Ar-H). MS m/z: $243\left(\mathrm{M}^{+}\right)$. Anal. Calcd. For $\mathrm{C}_{11} \mathrm{H}_{9} \mathrm{~N}_{5} \mathrm{~S}: \mathrm{C}, 54.32 ; \mathrm{H}, 3.70 ; \mathrm{N}$, 28.80. Found: C, 54.30; H, 3.72; N, 28.78.

2-Hydrazino-9-methyl[1,3,4]thiadiazepino[7,6-b]quinoline (2b). Prepared from methyl derivative of quinoline $\mathbf{1 b}(0.004 \mathrm{mmol})$ and carbidimide $(0.005 \mathrm{mmol})$. The compound obtained as a greenish yellow coloured solid after recrystalisation from aqueous DMF. Irradiation time: 4 minutes, Yield: 92\% (MW), refluxed time 5.5 hours, 72\% (Conventional). Mp: $180-182{ }^{\circ} \mathrm{C}$. IR $(\mathrm{KBr}) \mathrm{cm}^{-1}$ 3345, 3160. 3305. ${ }^{1} \mathrm{H}$ NMR (400 MHz, DMSO-d $\left.\mathrm{d}_{6}\right) 4.5$ (s, 2H, NH$), 8.1(\mathrm{~s}, 1 \mathrm{H}, \mathrm{NH})$, 7.1-8.1 (m, 5H, Ar-H). MS m/z: $257\left(\mathrm{M}^{+}\right)$. Anal. Calcd. For $\mathrm{C}_{12} \mathrm{H}_{11} \mathrm{~N}_{5} \mathrm{~S}: \mathrm{C}, 56.03 ; \mathrm{H}, 4.28 ; \mathrm{N}$, 27.23. Found: C, 56.00; H, 4.25; N, 27.26.

2-Hydrazino-8-methyl[1,3,4]thiadiazepino[7,6-b]quinoline (2c). Prepared from methyl derivative of quinoline $1 \mathrm{c}(0.004 \mathrm{mmol})$ and carbidimide $(0.005 \mathrm{mmol})$. The compound obtained as a greenish yellow coloured solid after recrystalisation from aqueous DMF. Irradiation time: 4 minutes,. Yield: 92\% (MW), refluxed time 6 hours, 70\% (Conventional). Mp: 187-189 ${ }^{\circ} \mathrm{C}$. IR $(\mathrm{KBr}) \mathrm{cm}^{-1} 3345,3160,3300 .{ }^{1} \mathrm{H}$ NMR (400 MHz, DMSO-d 6 ) 4.6 (s, 2H, NH ), 8.2 (s, 1H, NH), 7.1-8.1 (m, 5H, Ar-H). MS m/z: $257\left(\mathrm{M}^{+}\right)$. Anal. Calcd. For $\mathrm{C}_{12} \mathrm{H}_{11} \mathrm{~N}_{5} \mathrm{~S}: \mathrm{C}, 56.03 ; \mathrm{H}, 4.28 ; \mathrm{N}$, 27.23. Found: C, 56.07; H, 4.29; N, 27.27.

2-Hydrazino-10-methyl[1,3,4]thiadiazepino[7,6-b]quinoline (2d). Prepared from methyl derivative of quinoline $1 \mathrm{e}(0.004 \mathrm{mmol})$ and carbidimide $(0.005 \mathrm{mmol})$. The compound obtained as a greenish yellow coloured solid after recrystalisation from aqueous DMF. Irradiation time: 4 minutes, Yield: 92\% (MW), refluxed time 6 hours, 67\% (Conventional). Mp: 194-196 ${ }^{\circ} \mathrm{C}$. IR $(\mathrm{KBr}) \mathrm{cm}^{-1}$ 3350, 3165. 3300. ${ }^{1} \mathrm{H}$ NMR (400 MHz, DMSO-d 6 ) 4.6 (s, 2H, NH ), 8.2 (s, 1H, NH), 7.1-8.1 (m, 5H, Ar-H). MS m/z: $257\left(\mathrm{M}^{+}\right)$. Anal. Calcd. For $\mathrm{C}_{12} \mathrm{H}_{11} \mathrm{~N}_{5} \mathrm{~S}: \mathrm{C}, 56.03 ; \mathrm{H}, 4.28 ; \mathrm{N}$, 27.23. Found: C, 56.05; H, 4.24; N, 27.24. 
8-Chloro-2-hydrazino[1,3,4]thiadiazepino[7,6-b]quinoline (2e). Prepared from chloro derivative of quinoline $\mathbf{1 f}(0.004 \mathrm{mmol})$ and carbidimide $(0.005 \mathrm{mmol})$. The compound obtained as a greenish yellow coloured solid after recrystalisation from aqueous DMF. Irradiation time: 4 minutes, Yield: 92\% (MW), refluxed time 6 hours, 69\% (Conventional). Mp: $215-217{ }^{\circ} \mathrm{C}$. IR $(\mathrm{KBr}) \mathrm{cm}^{-1}$ 3340, 3150. 3310. ${ }^{1} \mathrm{H}$ NMR (400 MHz, DMSO-d $) 4.7$ (s, 2H, NH ), 8.2 (s, 1H, NH), 7.1-8.1 (m, 5H, Ar-H). MS m/z: $277\left(\mathrm{M}^{+}\right)$. Anal. Calcd. For $\mathrm{C}_{11} \mathrm{H}_{8} \mathrm{~N}_{5} \mathrm{ClS}: \mathrm{C}, 47.65 ; \mathrm{H}, 2.88 ; \mathrm{N}$, 25.27. Found: C, 47.63; N, 25.24; H, 2.52.

2-Hydrazino-9-methoxy[1,3,4]thiadiazepino[7,6-b]quinoline (2f). Prepared from methoxy derivative of quinoline $\mathbf{1 g}(0.004 \mathrm{mmol})$ and carbidimide $(0.005 \mathrm{mmol})$. The compound obtained as a greenish yellow coloured solid after recrystalisation from aqueous DMF. Irradiation time: 4 minutes, Yield: 93\% (MW), refluxed time 5.5 hours, 66\% (Conventional). Mp: $169-171{ }^{\circ} \mathrm{C}$. IR $(\mathrm{KBr}) \mathrm{cm}^{-1} 3340,3160,3310 .{ }^{1} \mathrm{H}$ NMR (400 MHz, DMSO-d $) 4.6$ (s, 2H, NH ), 8.2 (s, 1H, NH), 6.9-7.9 (m, 5H, Ar-H). MS m/z: $273\left(\mathrm{M}^{+}\right)$. Anal. Calcd. For $\mathrm{C}_{12} \mathrm{H}_{11} \mathrm{~N}_{5} \mathrm{OS}: \mathrm{C}, 52.74 ; \mathrm{H}, 4.03 ; \mathrm{N}$, 25.64. Found: C, 52.72; H, 4.06; N, 25.66.

2-Hydrazino-10-methoxy[1,3,4] thiadiazepino[7,6-b]quinoline (2g). Prepared from methoxy derivative of quinoline $\mathbf{1 h}(0.004 \mathrm{mmol})$ and carbidimide $(0.005 \mathrm{mmol})$. The compound obtained as a greenish yellow coloured solid after recrystalisation from aqueous DMF. Irradiation time: 4 minutes, Yield: 93\% (MW), refluxed time 6 hours, 71\% (Conventional). Mp: 198-200 ${ }^{\circ} \mathrm{C}$. IR $(\mathrm{KBr}) \mathrm{cm}^{-1} 3335,3150,3300 .{ }^{1} \mathrm{H}$ NMR (400 MHz, DMSO-d $) 4.6$ (s, 2H, NH 2$), 8.2$ (s, 1H, NH), 6.9-7.9 (m, 5H, Ar-H). MS m/z: $273\left(\mathrm{M}^{+}\right)$. Anal. Calcd. For $\mathrm{C}_{12} \mathrm{H}_{11} \mathrm{~N}_{5} \mathrm{OS}$ : C, 52.74; H, 4.03; N, 25.64. Found: C, 52.76; H, 4.08; N, 25.68.

2-Hydrazino-8-methoxy[1,3,4]thiadiazepino[7,6-b]quinoline (2h). Prepared from methoxy derivative of quinoline $\mathbf{1 b}(0.004 \mathrm{mmol})$ and carbidimide $(0.005 \mathrm{mmol})$. The compound obtained as a greenish yellow coloured solid after recrystalisation from aqueous DMF. Irradiation time: 4 minutes, Yield: 93\% (MW), refluxed time 5.5 hours, 71\% (Conventional). Mp: $164-166{ }^{\circ} \mathrm{C}$. IR $(\mathrm{KBr}) \mathrm{cm}^{-1}$ 3340, 3145, 3305. ${ }^{1} \mathrm{H}$ NMR (400 MHz, DMSO-d 6 ) $4.6\left(\mathrm{~s}, 2 \mathrm{H}, \mathrm{NH}_{2}\right), 8.2(\mathrm{~s}, 1 \mathrm{H}, \mathrm{NH})$, 6.9-7.9 (m, 5H, Ar-H). MS m/z: $273\left(\mathrm{M}^{+}\right)$. Anal. Calcd. For $\mathrm{C}_{12} \mathrm{H}_{11} \mathrm{~N}_{5} \mathrm{OS}: \mathrm{C}, 52.74 ; \mathrm{H}, 4.03 ; \mathrm{N}$, 25.64. Found: C, 52.75; H, 4.05; N, 25.62.

\section{Acknowledgements}

Authors are thankful to Indian Institute of Science, Bangalore for Spectral data. 


\section{References}

1. (a) Luzzio, M. J.; Besterman, J. M.; Emerson, D. L.; Evans, M. G.; Lackey K, P.; Leitner, L.; McIntyre, G.; Morton, B.; Myers, P. L.; Peel, M.; Sisco, J. M.; Sternbach, D. D.; Tong, W. Q.; Truesdale, A. D.; Uehling, E.; Vuong, A.; Yates, J. J. Med. Chem. 1995, 38, 395. (b) Wall, M. E.; Wani, M. C.; Nicholas, A. W.; Manikumar, G.; Tele, C.; Moore, A.; Truesdale, D.; Leitner, P.; Besterman, J. M. J. Med. Chem. 1993, 36, 2689.

2. Gatto, B.; Capranico, G.; Palumbo, M. Curr. Pharacol. Design 1999, 5, 195.

3. (a) Rewcastle, G. W.; Atwell, G. J.; Chambers, D. B.; Gaguley, C.; Denny, W. A, J. Med. Chem. 1986, 29, 472.

4. (a) Gopal, M.; Veeranna, S.; Doddamani, L. S. Spect Lett. 2004, 37, 347. (b) M. Gopal, M.; Shenoy, S.; Doddamani, L. S. J. Photochem. Photobiol. 2003, 72, 69.

5. Loaiza, P. R.; Quintero, A.; Rodríguez-Sotres, R. Solano, J. D.; Rocha, A. L. Eur. J. Med. Chem. 2004, 39, 5.

6. Althuis, T. H.; Khadin, S. B.; Czuba, L. J.; Moore, P. F.; Hess, H. J.; J. Med. Chem. 1980, $23,262$.

7. Jiang J. B.; Isaacson, D. U S Patent. 1987, 4656274.

8. Graeve, R. E.; Pociask J. R.; and Stein, R. G.; U S Patent. 1971, 3600393.

9. Farghaly, A. M.; Habib, N. S.; Khalil, M. A.; El-Sayed O. A.; Alaxandria, A. J. Pharm. Sci. 1989, 3, 90.

10. Zikan, V.; Radl, S.; Smejkal, F.; Zelena, D. Czech. Patent. 1986, 233445; Chem. Abstr. 1987, 106, 138447.

11. Demirbas, N.; Demirbas, A.; Karaoglu, S. A.; Çelik, E. Arkivoc 2005, (i), 75.

12. Kritsanida, M.; Mouroutsou, A.; Marakos, P.; Pouli, N.; Papakonstantinou Garoufalias, S.; Pannecouque, C.; Witvrouw, M.; De Clercq, E. Il Farmaco 2002, 57, 253.(b) Gururaja, R.; Jyoti, C. H.;. Vagdevi, H. M.; Kalluraya, B. I. J. Het. Chem 2004, 14, 97.

13. Mohan, J.; Anjaneyulug, G. S. R. Curr. Sci. 1989, 58, 1028.

14. Omar, A. M. M. E.; Aboulwafa, O. M. J. Heterocyclic. Chem. 1986, 23, 1339.

15. Prasad, A. R.; Ramalingamat, T.; Rao, A. B.; Diwan, P. W.; Sattur, P. B. Indian J. Chem. 1986, 25B, 556.

16. Deshmukh, A. A.; Mody. M. K.; Ramalingamat, T.; Sattur, P. B. Indian J. Chem. 1985, 25B, 793.

17. Invidiata, F. P.; Grimaudo, S.; Giammanco, P.; Giammanco, L. Farmaco 1991, 46, 1489.

18. Forest, M. C.; Lahouratate, P.; Martin, M.; Nadler, G.; Quiniou, M. J.; Zimmermann, R. G. J. Med. Chem. 1992, 35, 163. (b) Jadhav, P. K.; Daneker, W. F; Woerner, F. J. USP 5506355.

19. Natalya, N.; Volkova, Evgeniy, V.; Tarasov, Luc V. M., Suzanne, T.; Wim, D.; Vasiliy A. B. J. Chem. Soc., Perkin Trans. 1, 2002, 1574.

20. Caddick, S. Tetrahedron 1995, 51, 10403.

21. Galema, S. A. Chem. Soc. Rev. 1997, 26.

22. Varma, R. S.; J. Heterocyclic Chem. 1999, 35, 1565. 
23. Katritzky, A. R.; Sandeep, K. S. Arkivoc 2003, (xiii), 68.

24. (a). Mahajan, M. P.; Sondhi, S. M.; Ralhan, N. K. Aust. J. Chem .1977, 30, 2057. (b) Sondhi, S. M.; Mahajan, M. P.; Ganda, A. K.; Ralhan, N. K. J. Indian Chem. Soc. 1978, 16B, 433. (c). Molina, P. J.; Lindon, A.; Tarraga. Tetrahedron 1994, 50 (33), 10029.

25. Krasovsky, A. N.; Kochergin Roman, P. M.; Khim, A. B. Geterotsikl. Soedin. 1976, 6, 856.

26. (a) Hesek, D.; Rybar, A.; Monatsh. Chem. 1994, 125(11), (b) Schulze, W.; Letsch, G. Pharmazie 1973, 28, 367. (c). Sammers, L. A.; Angew. Chem. 1966, 78, 644.

27. (a) Donia, R. A.; Shotton, J. A.; Bentz, L. O.; Smith, G. E. P.; J. Org. Chem. 1949, 14, 952. (b) Spry, D. O.; Bhala, A. R.; Spitzer, W. A.; Jones, N. D.; Swartzendruber, J. K. Tetrahedron Lett. 1984, 25, 2531. (c). Corriu, R. J. P.; Lanneau, G. F.; Mehta, V. D. J. Organomet. Chem. 1991, 419, 9.

28. (a) Raghavendra, M.; Bhojya Naik, H. S.; Sherigara, B. S. J. Sulfur Chem. 2006, 27, 347. (b) Nandeshwarappa, B. P.; Arun Kumar, D. B.; Bhojya Naik, H. S.; Vaidya, V. P.; Mahadevan, K. M. J. Sulfur. Chem. 2005, 26, 373. (c) Bhojya Naik, H. S.; Ramesh, M. S.; Swetha, B. V.; Roopa T. R. Phos. Sulf. Sil. Reltd. Elem. 2006, 181, 533 (d) Nandeshwarappa, B. P.; Arun Kumar, D. B.; Bhojya Naik, H. S.; Vaidya, V. P.; Mahadevan, K. M.. Indian J. Chem. 2005, $44 B, 2155$.

29. Meth-Cohn, O.; Narine, B.; Tarnowski, B. J. Chem. Soc. Perkin Trans. 1981, 1, 1520. 